\title{
Electron density contour maps via Rietveld-MEM analysis using HR-XRD for the polycrystalline ferroelectric $B C Z T$
}

\author{
G. Herrera-Pérez ${ }^{a}$, J. Plaisier ${ }^{b}$, A. Reyes-Rojas ${ }^{c}$, and L. Fuentes-Cobas ${ }^{c}$ \\ ${ }^{a}$ Catedra CONACyT assigned to Centro de Investigación en Materiales Avanzados S. C. \\ Miguel de Cervantes 120, Chih., Chihuahua 31136, México. \\ ${ }^{b}$ Elettra-Sincrotrone Triestre S. C. p. A, \\ Strada Statale 14, Km 163.5 Bassovizza, Trieste 34149, Italy. \\ ${ }^{c}$ Physics of Materials Department. Centro de Investigación en Materiales Avanzados S. C. \\ Miguel de Cervantes 120, Chih., Chihuahua 31136, México.
}

Received 11 October 2021; accepted 15 November 2021

\begin{abstract}
The maximum entropy method in combination with the Rietveld refinement method applied to the analysis (Rietveld-MEM analysis) of high-resolution x-ray diffraction (HR-XRD) is an important tool to elucidate the electron density distribution and chemical bonding nature of materials. In this work, we present the comparison of electron density distribution obtained from the Rietveld-MEM analysis for polycrystalline perovskite $\mathrm{BaTiO}_{3}$ (reference sample) and $\mathrm{Ba}_{0.8} \mathrm{Ca}_{0.2} \mathrm{Zr}_{0.1} \mathrm{Ti}_{0.9} \mathrm{O}_{3}(B C Z T)$ ). To perform this task, HR-XRD patterns using synchrotron radiation were acquired. Tetragonal phase with $\mathrm{P} 4 \mathrm{~mm}$ (No.99) space group and pseudo-Voigt function were considered to model the HR-XRD peaks by the Rietveld method using the profile fitting Fullprof suite program. VESTA software was used to visualize 3D, 2D electron density distribution maps and line profiled to monitor the chemical bonding nature between Ba-O and Ti-O interactions and to visualize the off-center displacement of $\mathrm{Ti}$ cations by the incorporation of $\mathrm{Zr}$ and $\mathrm{Ca}$ cations. The interaction between $\mathrm{Ti}$ contours with $\mathrm{O}$ contours in the electron density distribution and the minimum electron density values revealed the enhancement of covalent nature and predominant ionic nature between barium and oxygen ions in the $B C Z T$. To monitor the ferroelectric hysteresis behavior, polarization versus electric field curves complement the characterization of these samples.
\end{abstract}

Keywords: XRD, ceramics, BCZT, electron density.

DOI: https://doi.org/10.31349/SuplRevMexFis.3.010601

\section{Introduction}

The electron density distribution of materials, in particular for ceramics, using high-resolution x-ray diffraction (XRD) patterns is an important tool to elucidate the bonding nature and the distribution of electrons in the bonding region (covalent or ionic character) that could be associated with a specific physical property (for example ferroelectricity in electro-ceramics). The Rietveld refinement [1] method applied to analyze XRD patterns is frequently performed by the use of the profile-fitting Fullprof Suite program [2]. The first program models the diffraction peaks in order to provide the structural information of materials such as the unit cell parameters or the atomic positions. The second program allows the visualization of the XRD pattern refined. The Fullprof Suite program has the advantage to perform a conventional Fourier analysis, and it is possible to reconstruct and to visualize the $2 \mathrm{D}$ and $3 \mathrm{D}$ electron contour maps using the GFourier program. To our knowledge, there is an alternative option, the statistical approach of Maximum Entropy Method (MEM) introduced in x-ray crystallography $[3,4]$ and implemented in VESTA software [5], which complements the electron density distribution that reveals the chemical bonding for materials. This method maximizes the initial electron density distribution information derived from the Rietveld refinement of XRD patterns resulting in a high resolved and more accurate electron density distribution than inverse Fourier trans- form. Previous works, in particular for the polycrystalline $\mathrm{BaTiO}_{3}$ and related compositions [6-8], suggested that MEM can be also used to determine the mid bond electron density, the integrated atomic charges, and the nature of the structural disorder.

In this work, polycrystalline $\mathrm{BaTiO}_{3}$ and $\mathrm{Ba}_{0.8} \mathrm{Ca}_{0.2} \mathrm{Zr}_{0.1} \mathrm{Ti}_{0.9} \mathrm{O}_{3}$ (denoted in this work as $B C Z T$ ) samples are chosen as they are well known lead-free electroceramics. These compositions are based on a perovskite structure with the $\mathrm{ABO}_{3}$ formula. The purpose of substituting $\mathrm{Ba}^{2+}$ by the homo-valent $\mathrm{Ca}^{2+}$ and $\mathrm{Ti}^{4+}$ by $\mathrm{Zr}^{4+}$ cations in the sites A and B respectively, is to favor the tetragonal phase and suppress the dielectric losses [9]. Furthermore, the high piezoelectric coefficient $d_{33}=690 \mathrm{pC} / \mathrm{N}$ [10] is attributed to $\mathrm{Zr}$ substitution due to $\mathrm{Zr}^{4+}$ possesses higher chemical stability than $\mathrm{Ti}^{4+}$. The combination of these results extends the potential applications for this electroceramic in the ceramic industry for example as a multilayer ceramic capacitor [9]. According to Kwei et al., [11], the tetragonal phase considers the ionic displacements of $\mathrm{Ti}$ and $\mathrm{O}$. The use of Rietveld refinement of high-resolution XRD patterns allows one to monitor for example the effects of $\mathrm{Ti}$ off-center displacement when $\mathrm{Zr}$ and Ca cations are introduced in the host structure. At the same time, it is possible to monitor the electron density distribution. As a result, one can distinguish qualitatively the presence of covalent character between $\mathrm{Ti}$ and $\mathrm{O}$ ions with respect to the ionic character 
between $\mathrm{Ba}$ and $\mathrm{O}$ ions. The early one is associated with the spontaneous polarization in the BCZT. As a knowledge contribution no prior studies have examined the electron density distributions obtained by MEM for BCZT.

The motivation of this work is to apply the MEM to the refined HR-XRD patterns using the Rietveld method. The Rietveld-MEM analysis allows to explain the electron density distribution qualitatively and quantitatively for the $B C Z T$. Rietveld-MEM results support the hybridization between $\mathrm{Ti}$ states with $\mathrm{O}$ states that are associated with the covalent character presented in $B C Z T$. We evaluate $\mathrm{BaTiO}_{3}$ as a reference compound. Then, we will monitor the $B C Z T$ (prepared by the modified Pechini method) to understand the in uence of $\mathrm{Zr}$ and $\mathrm{Ca}$ concentrations in the off-center displacement of Ti cations and its tetragonality. For this purpose, we collected HR-XRD patterns using synchrotron radiation. Polarization versus electric field curves to evaluate the hysteresis loops complement the characterization for both compositions.

\section{Materials and Methods}

A detailed preparation procedure for obtaining the $B C Z T$ powders by the modified Pechini method has been described elsewhere [12]. The powders were pressed in pellet shape with a diameter of $1 \mathrm{~cm}$ and thickness of $2 \mathrm{~mm}$ applying a pressure of $10 \mathrm{ton} / \mathrm{cm}^{2}$. These green pellets were heattreated at $1400^{\circ} \mathrm{C}$ for $5 \mathrm{~h}$ with heating and cooling ramps of $5^{\circ} \mathrm{C} / \mathrm{min}$ to promote grain growth in the micron size. The sintered pellets were characterized by high-resolution x-ray diffraction patterns at room temperature. The HR-XRD patterns were collected at the beamline 7.1 MCX of Elettra sincrotrone with a $\lambda=0.95 \AA$ and full width half maximum $(\mathrm{FWHM})=0.016-0.040^{\circ}$. Lattice parameters and other structural parameters of the $B C Z T$ phase were determined by the Rietveld refinement method using the profile fitting Fullprof Suite program version 2019 [2]. The model considered a tetragonal phase with $P 4 \mathrm{~mm}$ space group taken from Kwei et al [11]. The fitting process involved scale factor, zero displacement correction, background coefficients, lattice parameters, pseudo-Voigt peak factor and reflections FWHM. The following step consisted in reconstructing the bi-dimensional and tri-dimensional electron density distribution using visualization for electronic and structural analysis (VESTA) program [5]. The electron densities were calculated by the Fourier transform of structure factors, which are calculated from structure parameters and atomic scattering factors of free atoms from the Rietveld refinements X-ray diffraction data. The special resolution of electron densities was $0.1 \AA$. To draw the contour lines in a two-dimensional image, the logarithmic model was computed. The line profile was obtained using the fractional coordinates $(\mathrm{x}, \mathrm{y}, \mathrm{z})$ of $\mathrm{Ba}-$ $\mathrm{O} 2$ and Ti-O2. Later on, before performing the polarizationelectric field (P-E) measurements to evaluate the hysteresis loops, Ag paste (ELCOAT, CANS, Korea) was applied on the BCZT pellets as electrodes. The samples were poled at $40 \mathrm{kV} / \mathrm{cm}$, in a silicone oil bath, for $30 \mathrm{~min}$ at $90^{\circ} \mathrm{C}$. The
P-E curves of the BCZT pellets were measured using a Radian Technologies Precision workstation (model P-WS) and TRE (model 609 A, Albuquerque, New Mexico, USA) and visualized with the VISION data acquisition software.

\section{Results and Discussion}

\subsection{Rietveld refinement of HR-XRD}

Figure 1 shows the comparison between HR-XRD patterns of $\mathrm{BaTiO}_{3}$ and $B C Z T$. It can be observed well-defined and sharp peaks that indicate the heat-treatment effect on the grain growth and high crystallinity of samples. Panel a) displays the HR-XRD ( $\left.Y_{\text {obs }}\right)$ labeled with the Miller indices for $\mathrm{BaTiO}_{3}$. The calculated pattern $\left(Y_{\text {calc }}\right)$, residual $\left(Y_{\text {obs }}-Y_{\text {calc }}\right)$ and Bragg positions are present in this figure. Table I shows the comparison of Rietveld refinement parameters such as lattice parameters, tetragonality, unit cell volume and reliability indices.

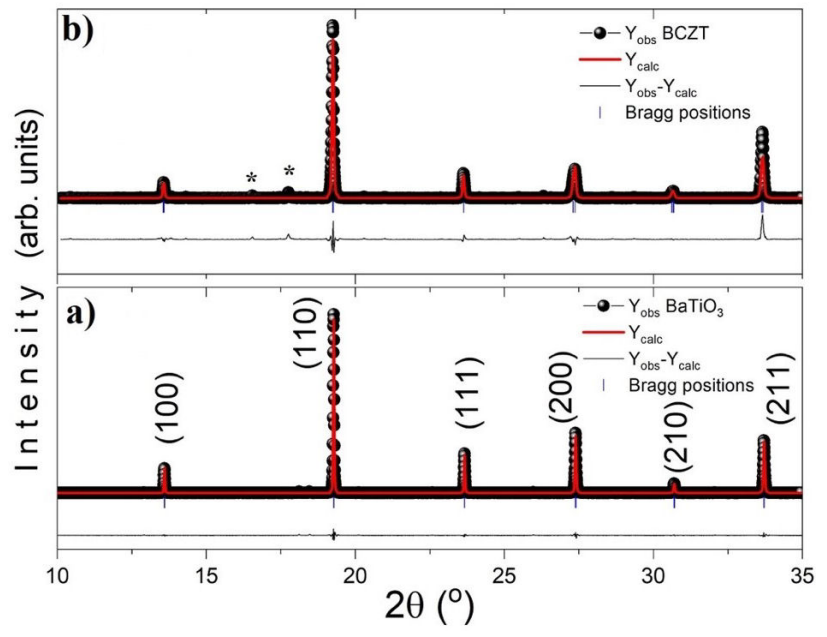

FIGURE 1. XRD pattern (black dotted line, $Y_{\text {obs }}$ ) for $B C Z T$ bulk $\mathrm{BaTiO}_{3}$ and $B C Z T$ sintered at $1400^{\circ} \mathrm{C}$ for $5 \mathrm{~h}$ and the comparison with refinement analysis (red solid line, $Y_{\text {calc }}$ ) by the Rietveld method using the profile fitting Fullprof Suite program. The horizontal (black solid line) represent the residual and the vertical lines (blue solid lines) are the Bragg positions.

TABLE I. Lattice parameters (a, b and c), tetragonality (c/a), volume, reliable-factors $\left(R_{p}, R_{w p}, R_{\exp }\right)$ and goodness-of-fit $\left(\chi^{2}\right)$.

\begin{tabular}{cccc}
\hline & Kwei et al., $[11]$ & $\mathrm{BaTiO}_{3}$ & $\mathrm{BCZT}$ \\
\hline $\mathrm{a}=\mathrm{b}(\AA)$ & $3.99095(29)$ & $3.9988(7)$ & $4.0080(9)$ \\
$\mathrm{c}(\AA)$ & $4.0352(3)$ & $4.0222(3)$ & $4.0136(1)$ \\
$\mathrm{c} / \mathrm{a}$ & 1.011 & 1.005 & 0.9986 \\
$\mathrm{~V}\left(\AA^{3}\right)$ & $64.271(14)$ & $64.316(12)$ & $64.478(33)$ \\
$R_{p}$ & -- & 4.93 & 10.9 \\
$R_{w} p$ & 4.55 & 6.53 & 15.4 \\
$R_{\exp }$ & 3.30 & 5.56 & 8.8 \\
$\chi^{2}$ & 2.8 & 1.4 & 2.9 \\
\hline
\end{tabular}



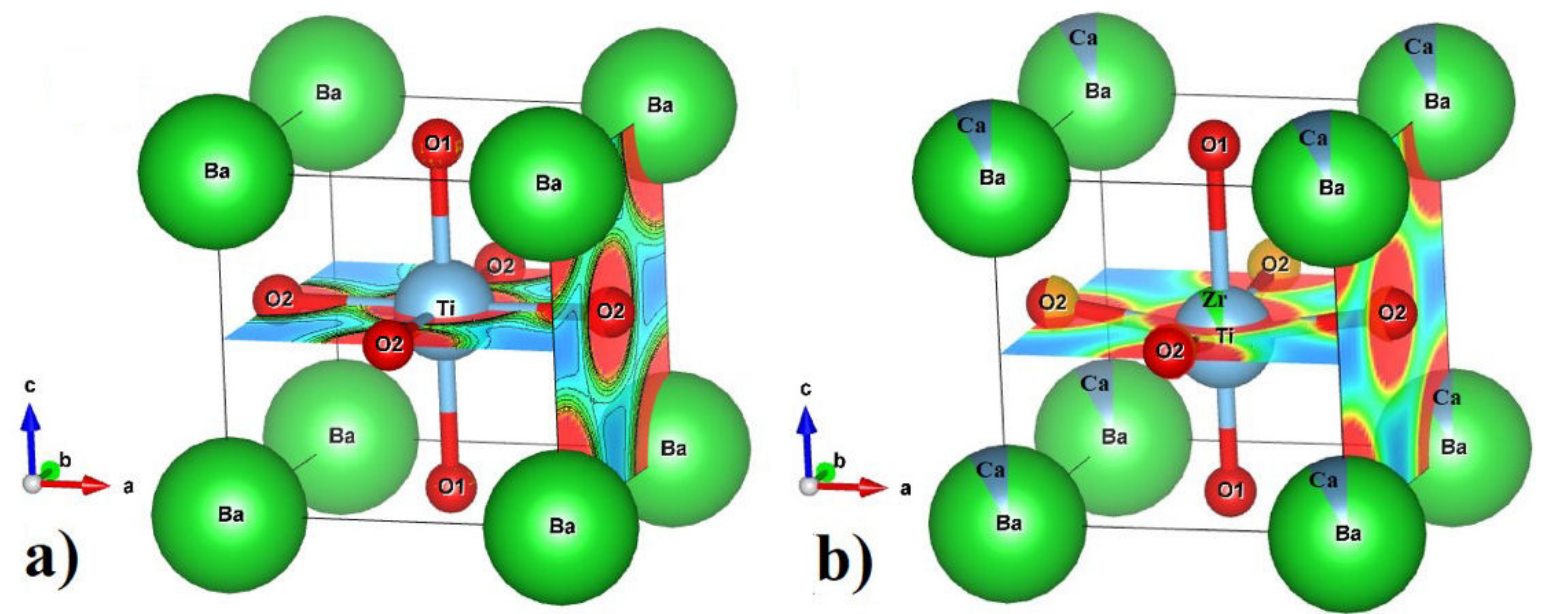

FIGURE 2. Three-dimensional unit cell with the electron density distribution maps at (100) and (001) plane for a) $\mathrm{BaTiO}_{3}$ and b) $B C Z T$.

TABLE II. One dimensional electron density variation along Ba-O2 and Ti-O2 bonds.

\begin{tabular}{|c|c|c|c|c|}
\hline sample & $\begin{array}{c}\text { Bond length } \\
\text { (Ba-O2) } \\
(\AA)\end{array}$ & $\begin{array}{c}\text { MED } \\
(\mathrm{Ba}-\mathrm{O} 2) \\
\left(e / \AA^{3}\right)\end{array}$ & $\begin{array}{c}\text { Bond length } \\
\text { (Ti-O2) } \\
(\AA)\end{array}$ & $\begin{array}{c}\text { MED } \\
(\mathrm{Ti}-\mathrm{O} 2) \\
\left(e / \AA^{3}\right)\end{array}$ \\
\hline \multicolumn{5}{|l|}{$\mathrm{BaTiO}_{3}$} \\
\hline Mangaiyarkarasi et al. [13] & 2.833 & 0.309 & 2.003 & 0.674 \\
\hline \multicolumn{5}{|l|}{$\mathrm{BaTiO}_{3}$} \\
\hline Sasikumar et al. [17] & 2.8567 & 0.3326 & 1.9268 & 0.7174 \\
\hline $\mathrm{BaTiO}_{3}$ (this work) & $2.7990(3)$ & $0.3442(5)$ & $1.9998(8)$ & $0.6424(8)$ \\
\hline$B C Z T$ (this work) & $2.7994(7)$ & $0.3946(9)$ & $2.0310(8)$ & $0.7402(6)$ \\
\hline
\end{tabular}

The tetragonal model proposed by Kwei et al. [11], led to a satisfactory result. The Rietveld refinement for HR-XRD $B C Z T$ is shown in panel b) The HR-XRD patterns confirm that all sintered samples exhibit polycrystalline perovskite structure in the tetragonal phase with $P 4 m m$ symmetry. The HR-XRD $B C Z T$ pattern shows two diffraction peaks that are identified by asterisk attributed to traces of $\mathrm{Ba}_{6} \mathrm{Ti}_{17} \mathrm{O}_{4} 0$ phase. The presence of this phase does not affect the ferroelectric properties as will be discussed.

\subsection{Electron density distribution by maximum entropy method}

The utilities of VESTA allow the reconstruction of the threedimensional electron density distribution maps around $\mathrm{Ba}, \mathrm{Ti}$, $\mathrm{O} 1$ and $\mathrm{O} 2$ ions in (100) and (001) planes (vertical and horizontal slices, respectively). Figure $2 \mathrm{a}$ ) and b) display the 3D view of the unit cell for $\mathrm{BaTiO}_{3}$ and $B C Z T$, respectively.

A color scale on each plane is displayed showing the high (red-colored area) and low (blue-colored area) electronic densities. As a first approach, in panel a) it can be noticed that the edge centers correspond to the electron densities of $\mathrm{Ba}$ atoms and in the middle of the (100) plane is located the O2, both with closed spherical contours followed by low electron density regions. The recognition of the chemical interaction type between $\mathrm{Ba}-\mathrm{O}$ contours is ionic. In comparison, $\mathrm{Ti}$ and $\mathrm{O}$ bonds exhibit shared-type contours that show a clear covalent bonding nature This result proved the $\mathrm{p}$-d overlap between $\mathrm{O}$ $2 \mathrm{p}$ and $\mathrm{Ti} 3 \mathrm{~d}$ orbitals. To obtain a more resolve electron density distribution one can visualize the two-dimensional contour image. Two-dimensional electron density distribution contour maps of the (100) plane are displayed in Fig. 3.

Panel (a) and (d) show $\mathrm{Ba}$ and $\mathrm{O}$ atoms with closedshell interactions (high-density regions) demonstrating the minimum interaction between them. This result confirms the predominant ionic character between barium and oxygen [13]. In contrast, share-type atomic interaction between $\mathrm{Ti}$ and $\mathrm{O}$ atoms in the (001) plane is observed in panels (b) and (e). This is a qualitative description of the covalent character as a result of hybridization between O $2 \mathrm{p}$ with Ti $3 \mathrm{~d}$ orbitals $[14,15]$. Moreover, it is possible to notice in panel (e) that the $\mathrm{Ti}$ and $\mathrm{O} 1$ atoms exhibit a large atomic displacement, which suggests the existence of distortions in agreement with Fig. 1b). From panels (c) and (f), one can observe the slice that corresponds to the (100) plane with an important hybridization due to the strong overlap between $\mathrm{O} 2 \mathrm{p}-\mathrm{Ti} 3 \mathrm{~d}$ orbitals. In this respect, Cohen and Krakauer [16] calculated and reported for the first time the electron density distribution for 


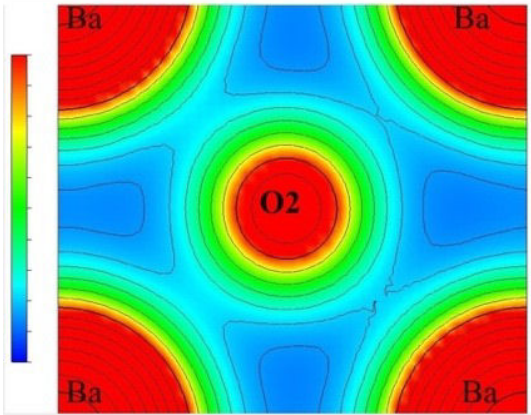

(a)

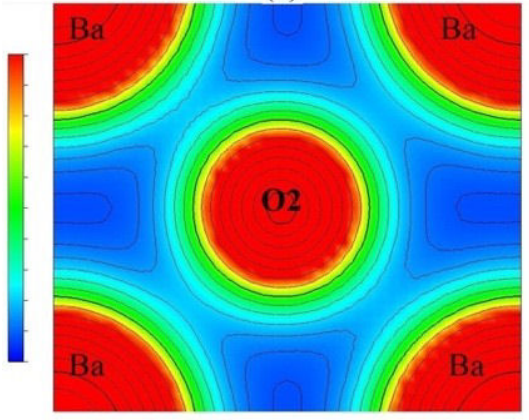

(d)

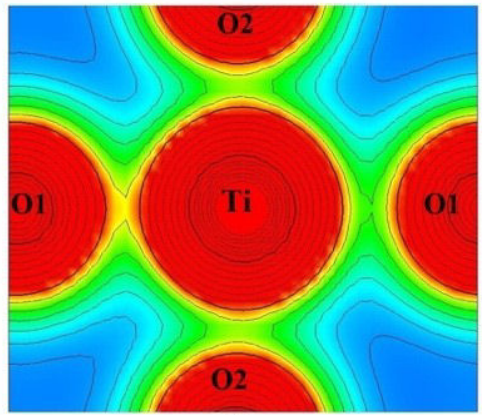

(b)

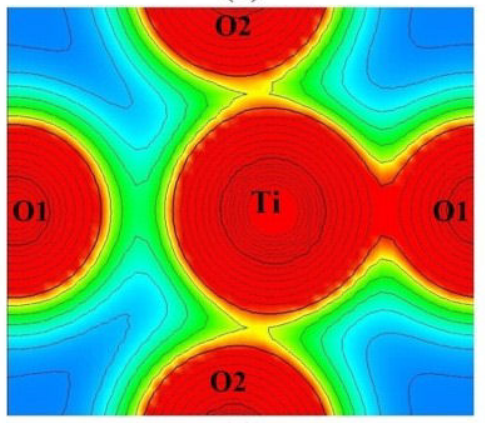

(e)

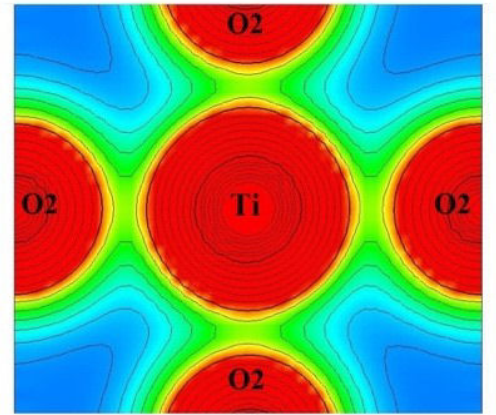

(c)

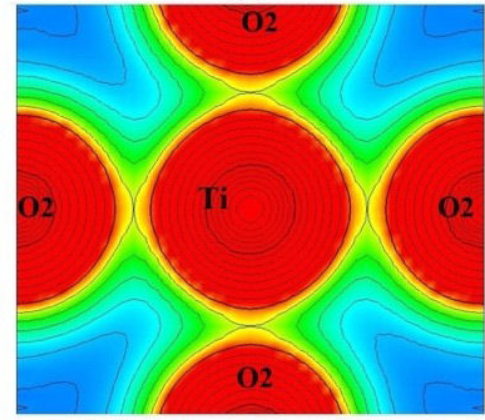

(f)

FIGURE 3. Comparison between the two-dimensional electron density distributions at (100), (100) and (001) planes for (a-c) BaTiO 3 (d-f) $B C Z T$.
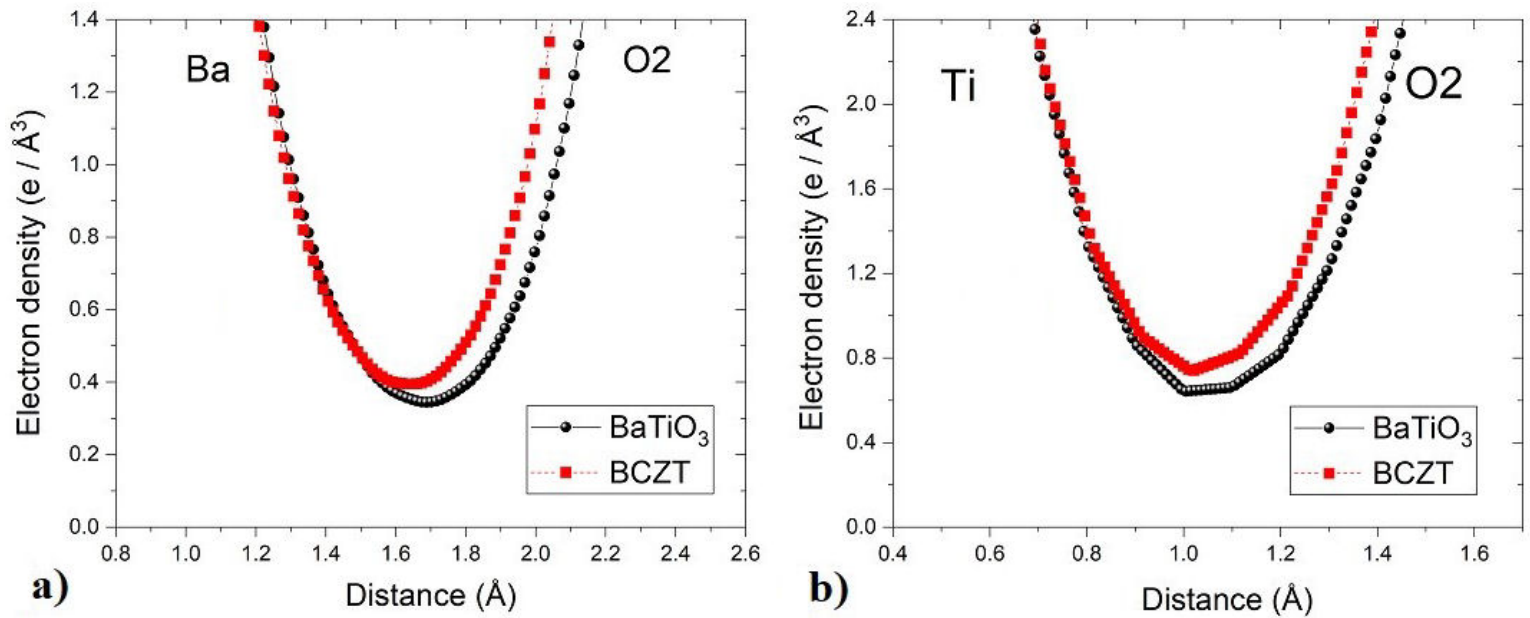

FIGURE 4. Comparison between line profiles for (a) Ba-O2 bonds and (b) Ti-O2 bonds.

$\mathrm{BaTiO}_{3}$. They suggest that covalent bonding has an influence on the spontaneous polarization of $\mathrm{BaTiO}_{3}$, and that the presence of $\mathrm{Ti}^{4+}$ and $\mathrm{Zr}^{4+}$ are favorable for ferroelectricity which tends to hybridize with $\mathrm{O} 2 \mathrm{p}$ states. To obtain a quantification that supports this qualitative description of the bonding nature, the line profile or one-dimensional electron density versus distance curves allows to analyze the chemical bonding in term of numerical values. In the next procedure, the position of minimum electron densities (MED) at a certain distance of Ba-O and Ti-O bond is reported.
The comparison between one-dimensional electron densities as a function of the distance of $\mathrm{BaTiO}_{3}$ (black circles with solid line) and $B C Z T$ (red square with dashed line) samples are shown in Fig. 4.

$\mathrm{Ba}-\mathrm{O} 2$ and $\mathrm{Ti}-\mathrm{O} 2$ bonds were selected to perform this comparison and they are displayed in panels a) and b), respectively. The values of MED also allow associating with the bonding nature in the samples. Higher MED values indicate larger covalency and a large covalency could cause spontaneous polarization [16-18]. Table II presents the comparison of bond length and the MED values at a certain distance for $\mathrm{BaTiO}_{3}$ and $\mathrm{BCZT}$. The bond lengths are found 


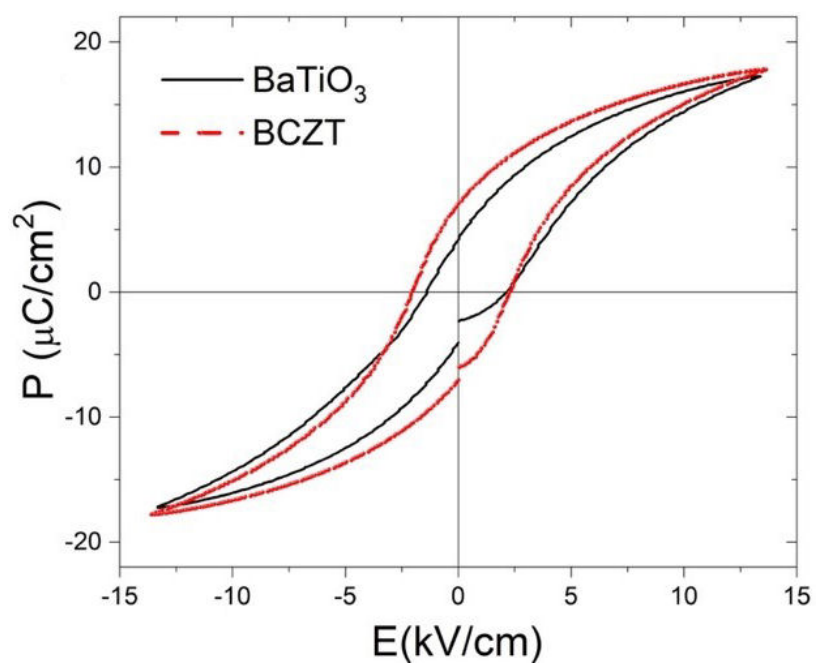

FIGURE 5. Polarization versus electric field curves that shows hysteresis loops for $\mathrm{BaTiO}_{3}$ (black solid line) and $B C Z T$ (red dashed line).

TABLE III. Ferroelectric properties, saturation polarization $\left(P_{s}\right)$, remnant polarization $\left(P_{r}\right)$, and coercive field $\left(H_{c}\right)$.

\begin{tabular}{ccc}
\hline & $\mathrm{BaTiO}_{3}$ & BCZT \\
\hline$P_{s}\left(\mu \mathrm{C} / \mathrm{cm}^{2}\right)$ & 17.3 & 17.3 \\
$P_{r}\left(\mu \mathrm{C} / \mathrm{cm}^{2}\right)$ & 4.2 & 7.1 \\
$H_{c}(\mathrm{kV} / \mathrm{cm})$ & 2.1 & 2.2 \\
\hline
\end{tabular}

to be increasing due to the incorporation of $\mathrm{Zr}^{4+}(1.34 \AA)$ in the $\mathrm{B}$-sites. As a reference, the mid bond density between $\mathrm{Ba}$ and $\mathrm{O} 2$ reported in the literature for $\mathrm{BaTiO}_{3}$ is $0.309 e / \AA^{3}$, which suggests a predominant ionic character [13]. The mid bond density between the Ti-O2 bond founded in the literature is $0.674 e / \AA^{3}[13]$. The mid bond for the $B C Z T$ shows an enhancement of ionic character for $\mathrm{Ba}-\mathrm{O} 2$ and covalent character for Ti-O2.

\subsection{Ferroelectric properties}

The polarization versus electric field curves obtained with a low electrical field and $1 \mathrm{~Hz}$ applied over $\mathrm{BaTiO}_{3}$ and $B C Z T$ pellets are display in Fig. 5. One can observe a clear hysteresis loop that is associated with the expected ferroelectric nature of the samples. The loop becomes saturated with an increasing electric field. Then, in these hysteresis loops were identified the saturation polarization $P_{s}$, remnant polarization $P_{r}$ and coercive field $H_{c}$ parameters.

These ferroelectric parameters are summarized in Table III. Moreover, from energy storage consideration, the charge storage density $Q_{c}$ can be determined by $Q_{c}=\left(P_{s}-\right.$ $\left.P_{r}\right)$. At $13 \mathrm{kV} / \mathrm{cm}, Q_{c}=10.7 \mu \mathrm{C} / \mathrm{cm}^{2}$. The total energy density of $B C Z T$ ceramics can be obtained by integrating the area between the polarization axis and the hysteresis loops. At $13 \mathrm{kV} / \mathrm{cm}$, the obtained total energy density is 0.05 $\mathrm{J} / \mathrm{cm}^{3}$. The presence of $\mathrm{Ba}_{6} \mathrm{Ti}_{17} \mathrm{O}_{40}$ may not influence in the $B C Z T$ hysteresis loops due that there is no evidence of a ferroelectric response [19].

\section{Conclusions}

In this work, we compared the chemical bonding nature and the ferroelectric properties for perovskite $\mathrm{BaTiO}_{3}$ and $\mathrm{Ba}_{0.8} \mathrm{Ca}_{0.2} \mathrm{Zr}_{0.1} \mathrm{Ti}_{0.9} \mathrm{O}_{3}(B C Z T)$ samples. In particular, we focus our attention to monitor the electronic distribution using the Rietveld-MEM analysis in HRXRD patterns. The samples were prepared by the modified Pechini method and sintered at $1400^{\circ} \mathrm{C}$ for $5 \mathrm{~h}$ to promote grain growth. In the first part of the present work, HR-XRD patterns using synchrotron radiation at the Elettra sincrotrone were acquired to monitor the evolution of lattice parameters and their tetragonality. The refinement of HR-XRD patterns was performed by the Rietveld method using the profile fitting Fullprof suite program. All refinements were performed considering a perovskite structure in tetragonal phase with $P 4 \mathrm{~mm}$ space group. A decrease of tetragonality was observed in the sample of $B C Z T$ with a slight increase of unit cell volume due to the $\mathrm{Zr}$ concentration. The second part consisted in elucidate the electron density distribution maps determined by the maximum entropy method using the VESTA software. The three and two-dimensional reconstruction of electron density distribution maps revealed the enhancement of interaction between contours of titanium with the contour of oxygen ions as the $\mathrm{Ca}$ concentration is present. This hybridization between Ti $3 \mathrm{~d}$ states with $\mathrm{O} 2 \mathrm{p}$ states supports that the chemical bonding covalent character plays an important role in this electroceramic. The mid-bond values from the line profile corroborate our interpretation of the bonding nature for the Ti-O interaction (covalent) and the Ba-O interaction (ionic). In addition, Polarization versus electric field curves show hysteresis loops demonstrating the ferroelectric behavior of $\mathrm{BaTiO}_{3}$ and $B C Z T$.
1. H.M. Rietveld, A profile refinement method for nuclear and magnetic structures, J. Appl. Crystallogr. 2 (1969) 65, https : //doi.org/10.1107/S0021889869006558

2. J. Rodriguez-Carbajal, Recent advances in magnetic structure determination by neutron powder diffraction. Physica B: Condensed Matter. 192 (1993) 55, https://doi.org/10.

\section{$1016 / 0921-4526(93) 90108-I$}

3. D.M. Collins, Electron density images from imperfect data by iterative entropy maximization. Nature 298 (1982) 49, https : //doi.org/10.1038/298049a0

4. M. Sakata, and M. Sato. Accurate structure analysis by the 
maximum-entropy method. Acta Cryst. A 46 (1990) 263, https://doi.org/10.1107/S0108767389012377

5. K. Momma, and F.Izumi, VESTA 3 for three-dimensional visualization of crystal, volumetric and morphology data. $J$. Appl. Crystallogr. 44 (2011) 1271, https : / / doi.org/10. 1107/S0021889811038970.

6. A. Rother et al., X-ray investigation, high-resolution electron holography, and density functional calculations of singlecrystalline $\mathrm{BaTiO}_{3}$. Phys. Rev. B. 74 (2006) 134116, https: //doi.org/10.1103/PhysRevB.74.134116

7. S. Sasikumar, et al., Effect of neodymium doping in $\mathrm{BaTiO}_{3}$ ceramics on structural and ferroelectric properties. J. Mater. Sci: Mater. Electron. 31 (2020) 1535, https://doi.org/ 10.1007/s10854-019-02670-6

8. I. B. Catellani et al., Study of the $\mathrm{BaTiO}_{3}$ electronic structure using the maximum entropy method and density functional theory calculations. Integrated Ferroelectrics 174 (2016) 104, https://doi.org/10.1080/10584587.2016. 1192926

9. B. Garbarz-Glos, K. Bormanis, and D. Sitko, Effect of $\mathrm{Zr}^{4+}$ Doping on the Electrical Properties of $\mathrm{BaTiO}_{3}$ Ceramics. Ferroelectrics 417 (2011) 118, https://doi.org/10. $1080 / 00150193.2011 .578508$.

10. W. Liu, and X. Ren, Large Piezoelectric Effect in Pb-Free Ceramics. Phys. Rev. Lett. 103 (2009) 257602, https : / doi. org/10.1103/PhysRevLett.103.257602.

11. G. H. Kwei, A. C. Lawson, S. J. L. Billinge, and S. W. Cheong, Structures of the ferroelectric phases of barium titanate. J. Phys. Chem. 97 (1993) 2368, https://doi.org/ $10.1021 / j 100112 a 043$

12. K. Jiménez, and G. Herrera, Lead-free piezoelectric ceramics as candidates on the development of morphing winglets in aircrafts. J. Mater. Sci. En. A 8 (2018) 32, https : / / doi . org/ $10.17265 / 2161-6213 / 2018.1-2.005$
13. J. Mangaiyararasi, R. Saravanan, and M. M. Ismail, Chemical bonding and charge density distribution analysis of undoped and lanthanum doped barium titanate ceramics. $J$. Chem. Sci. 128 (2016) 1913, https://doi.org/10. 1007/s12039-016-1190-1.

14. E. Orhan et al., Room-temperature photoluminescence of $\mathrm{BaTiO}_{3}$ : Joint experimental and theoretical study. Phys. Rev. B 71 (2005) 085113, https://doi.org/10.1103/ PhysRevB.71.085113.

15. J. Mangaiyarkkarasi, S. Sasikumar, O. V. Saravanan, and R. Saravanan, Electronic structure and bonding interaction in $\mathrm{Ba}_{1-x} \mathrm{Sr}_{x} \mathrm{Zr}_{0.1} \mathrm{Ti}_{0.9} \mathrm{O}_{3}$ ceramics. Front. Mater. Sci. 11 (2017) 182, https://doi.org/10.1007/ s11706-017-0376-x

16. R. E. Cohen, and H. Krakauer, Electronic structure studies of differences in ferroelectric behaviour of $\mathrm{BaTiO}_{3}$ and $\mathrm{PbTiO}_{3}$. Ferroelectrics 136 (1992) 65, https : / / doi.org/ $10.1080 / 00150199208016067$

17. S. Sasikumar, S. Saravanakumar, S. A. Bahadur, and D. Sivaganesh, Electronic structure, optical and chemical bonding properties of strontium doped Barium Titanate. Optik 206 (2020) 163752, https://doi.org/10.1016/j. ijleo.2019.163752

18. G. Herrera-Pérez et al., Multiplet structure for perovskite-type $\mathrm{Ba}_{0.9} \mathrm{Ca}_{0.1} \mathrm{Ti}_{0.9} \mathrm{Zr}_{0.1} \mathrm{O}_{3}$ by core-hole spectroscopies. J. Appl. Phys. 128 (2020) 064106, https: //doi.org/10.1063/ 5.0014496

19. H. Beltrán, B. Gómez, E. Masó Cordoncillo, P. Escribano, and A. R. West, Electrical properties of ferroelectric $\mathrm{BaTi}_{2} \mathrm{O}_{5}$ and dielectric $\mathrm{Ba}_{6} \mathrm{Ti}_{17} \mathrm{O}_{40}$ ceramics. J. Appl. Phys. 128 (2005) 064106, https://doi.org/10.1063/1.1862766 